\title{
Epitope profiling of coronavirus-binding antibodies using computational structural modelling
}

\author{
Sarah A. Robinson ${ }^{1 *}$, Matthew I. J. Raybould ${ }^{1 *}$, Constantin Schneider ${ }^{1}$, Wing Ki Wong ${ }^{1}$, Claire Marks ${ }^{1}$, and Charlotte M. \\ Deane $^{1, \bowtie}$ \\ ${ }^{1}$ Oxford Protein Informatics Group, Department of Statistics, University of Oxford, UK
}

\begin{abstract}
Identifying the epitope of an antibody is a key step in understanding its function and its potential as a therapeutic. It is wellestablished in the literature that sequence-based clonal clustering can identify antibodies with similar epitope complementarity. However, there is growing evidence that antibodies from markedly different lineages but with similar structures can engage the same epitope with near-identical binding modes. Here, we describe a novel computational method for epitope profiling based on structural modelling and clustering, and show how it can identify sequence-dissimilar antibodies that engage the same epitope. We start by searching for evidence of structural conservation across the latest solved SARS-CoV-2-binding antibody crystal structures. Despite the relatively small number of solved structures, we find numerous examples of sequencediverse but structurally-similar coronavirus-binding antibodies engaging the same epitope. We therefore developed a highthroughput structural modeling and clustering method to identify functionally-similar antibodies across the set of thousands of coronavirus-binding antibody sequences in the Coronavirus Antibody Database (CoV-AbDab). In the resulting multipleoccupancy structural clusters, $92 \%$ bind to consistent domains based on CoV-AbDab metadata. Our approach functionally links antibodies with distinct genetic lineages, species origins, and coronavirus specificities. This indicates greater convergence exists in the immune responses to coronaviruses than would be suggested by sequence-based approaches. Our results show that applying structural analytics to large class-specific antibody databases will enable high confidence structure-function relationships to be drawn, yielding new opportunities to identify functional convergence hitherto missed by sequence-only analysis.
\end{abstract}

structural clustering | coronavirus antibody | structure-function | repertoire profiling

${ }^{*}$ These authors contributed equally to this work.

凶Correspondence: deane@stats.ox.ac.uk

\section{Introduction}

The COVID-19 pandemic has generated worldwide efforts to isolate and characterise antibodies able to confer protection against SARS-CoV-2. Hundreds of studies have now released data on diverse antibodies and nanobodies capable of binding at least one coronavirus antigen (1).

Due to the escalating number of individuals infected by SARS-CoV-2, most of the reported coronavirus-binding antibodies to date have been sourced directly from the blood of convalescent human patients. The primary technique used to identify such binders is 'serum baiting', where an extracellular coronavirus antigen is used to pan donated blood serum directly for complementary antibodies $(2,3)$. Another increasingly-used method is deep sequencing of the SARS$\mathrm{CoV}-2$ convalescent $\mathrm{B}$-cell receptor (BCR) repertoire, which can implicate particular expanded antibody lineages as important to adaptive immunity without biasing towards a chosen antigen bait (4-6). Other discovery methods have included mining surface display libraries, challenging and harvesting transgenic animals, and antibody engineering (1).

As of 11th March 2021, over 2,400 SARS-CoV-2 binding antibodies and nanobodies had been identified, of which just under one-third show neutralisation activity against the virus. The properties (including sequence and, where possible, structure) of these antibodies are documented in the Coronavirus Antibody Database (CoV-AbDab), which tracks patents and the academic literature on a weekly basis (1). One way to use this collated data is to look for similarities between binders. For instance, when a novel antibody is antigen-baited out of SARS-CoV-2 response serum, or is identified as an expanded clonal lineage post-SARS-CoV2 infection, one can assess whether it bears resemblance to any other antibody previously reported to bind a coronavirus. This resemblance can then be used to predict functional properties of the newly-isolated antibody, such as its site of engagement with the antigen (the 'epitope').

A common way to cluster antibodies into such functional groupings is 'clonotyping', a form of clonal lineage clustering. This can be performed in several different ways (7). For example, strict Fv-clonotyping maps both VH and VL antibody chains to their closest immunoglobulin V- and J-gene and subsequently clusters identical gene mappings by their CDRH3 and CDRL3 lengths and sequence identities (using a threshold close to $100 \%$ per CDR3 region). This approach typically yields tight and functionally-significant clustering, but is severely limited by its ability to bring together all antibodies able to engage a particular epitope (8-10). As a result, leniency is often introduced to the clonotyping protocol, by lowering the sequence identity threshold to $80 \%$ (11), ignoring J-gene annotations, and/or only considering the heavy chain (VH-only clonotyping) (4).

Convergent lenient VH-only clonotypes have been identified between multiple SARS-CoV-2 infected or convalescent individuals (12-18) and across different studies, for example 
the overlap between the clonotypes found by Galson et al. (4) and Nielsen et al. (19). Several papers have compared $\mathrm{BCR}$ sequences from individuals to verified SARS-CoV-2 binders in CoV-Ab-Dab and identified clonal similarities (e.g. $(4,15))$.

Despite this, the clonotypes found to be enriched/to bear similarity to CoV-AbDab antibodies post SARS-CoV-2 exposure often differ across studies (20-22). This may be partly due to the small sample sizes used in individual studies, and the intrinsic biases in individual VJ gene usage; Xiang et al. found a larger variation between individuals within a cohort (healthy, or three different severities of COVID symptoms) than between cohorts (22). Another contributing factor is lineage clustering itself. Levels of functional convergence may in fact be higher than implied even by lenient clonotyping, as antibodies that derive from different lineages can engage the same epitope. The evidence for this phenomenon has been growing over recent years $(9,10,23-25)$. For example, solved structures of antibody-antigen complexes reveal pairs of antibodies with different genetic origins but sufficiently similar binding site geometry and paratope similarity that they bind to the same antigen with near-identical binding modes $(9,10)$. Furthermore, given that individuals' naive repertoires typically share very few clonotypes $(11,26)$ and yet are often found to respond to similar 'immunodominant' epitopes, it follows that multiple evolutionary routes may lead from low-moderate affinity naive BCRs to high affinity antibodies against the same antigen surface region. This is supported by statistical arguments showing the implausability of a purely "random repertoire" for an efficient immune response $(25,27)$. Epitope immunodominance could be rationalised via the existence of a more 'public' set of backbone structures in the BCR repertoire and the concept that BCRs with similar topologies and sufficient chemical complementarity engage the same epitope $(24,25)$.

Structural comparisons offer a way to analyse antibody data over and above clonotype-based approaches. Analysis of epitope regions using solved structures and competition assays of SARS-CoV-2 - binding antibodies has already revealed discrete antibody binding sites $(28,29)$. However, such assessments are very biased towards RBD-binding, neutralising antibodies, whose therapeutic potential renders them worth the expense and effort of structure determination. The vast majority of datapoints in CoV-AbDab do not have solved structures and must instead be structurally modeled. While homology models are provided alongside each structurallyunsolved CoV-AbDab entry, no studies have yet harnessed this data for functional annotation.

In this analysis, we examine how structurally analysing $\mathrm{CoV}-\mathrm{AbDab}$ can enhance our functional understanding of coronavirus-binding antibodies. We first analyse all X-ray crystal structures of antibodies/nanobodies bound to SARSCoV-2 antigens, showing both that structure is conserved more often than clonality across same-epitope binders, and that paratope profiles typically involve multiple regions of the antibody across both chains. This provides direct evidence that relatively sequence dissimilar coronavirus-binding antibodies with high variable domain $(\mathrm{Fv})$ structural similarity are able to exhibit functional commonality. We then model and structurally cluster the thousands of antibody $\mathrm{Fv}$ sequences in CoV-AbDab and show that $92 \%$ of multipleoccupancy structural clusters bin together antibodies that bind to consistent coronavirus antigens/domains. We also show that, in accordance with our analysis of the SARS-CoV$2 \mathrm{X}$-ray co-crystal structures, the antibodies within these structural clusters frequently transcend clonal lineages. This not only demonstrates that our computational structural analysis pipeline provides orthogonal information to clonotyping to improve antibody functional profiling, but also that antibody immune responses to SARS-CoV-2 are likely to be even more convergent than currently understood.

\section{Results}

Sequences and structures in CoV-Ab-Dab. The growth of coronavirus-binding antibody and nanobody data in CoVAbDab since its public release on $7^{\text {th }}$ May 2020 is shown in Figure 1. The antibody plot indicates how the availability of sequence data rose much more rapidly than structural data at the start of the pandemic, stabilising at a level roughly an order of magnitude higher. However, the availability of solved antibody structures increased markedly in October 2020 and has continued to grow at an even faster rate throughout 2021.

Analysis of SARS-CoV-2-antibody structural complexes. Experimentally-solved structures allow us to analyse the diversity of antibody geometries and paratopes that engage coronavirus antigens (28-30). At the time of this study (11 ${ }^{\text {st }}$ March 2021), CoV-AbDab (1) referenced 2,304 antibodies and 420 nanobodies able to bind to a coronavirus, with 132 having at least one solved structure. 111 of these solved structures are binders to SARS-CoV-2 (Figure 1), of which 91 were solved in complex with the cognate antigen.

A total of 48 antibodies and 12 nanobodies had at least one published solved X-ray crystal structure in complex with SARS-CoV-2 (12, 14, 20, 28, 31-54), all binding to the spike receptor binding domain (RBD, see Table S1 and Table S2 for names and PDB codes), while a further 31 antibodies and nanobodies were solely structurally characterised by cryoEM (1). In our analysis we have focused on the 60 crystal structures, in order to determine more precise antibody binding site topologies and paratope profiles.

Epitope Binning. Inspecting the 48 antibodies solved by Xray crystallography in complex with the RBD, 46 appear to fall cleanly into the binding regions previously defined by Dejnirattisai et al. (28) (only approximate as the original clustering was performed via competition assays). The two remaining antibodies spanned the left and right shoulder regions (see Table S1). Interestingly, most of the 12 nanobodies could also be assigned to these predefined regions (9/12, see Table S2).

Structural alignment of the RBD of all complexes reveals that the 22 antibodies that bind to the 'neck' cluster (as termed by Dejnirattisai et al. (28)) have high structural conserva- 
bioRxiv preprint doi: https://doi.org/10.1101/2021.04.12.439478; this version posted April 12, 2021. The copyright holder for this preprint (which was not certified by peer review) is the author/funder, who has granted bioRxiv a license to display the preprint in perpetuity. It is made available under aCC-BY 4.0 International license.
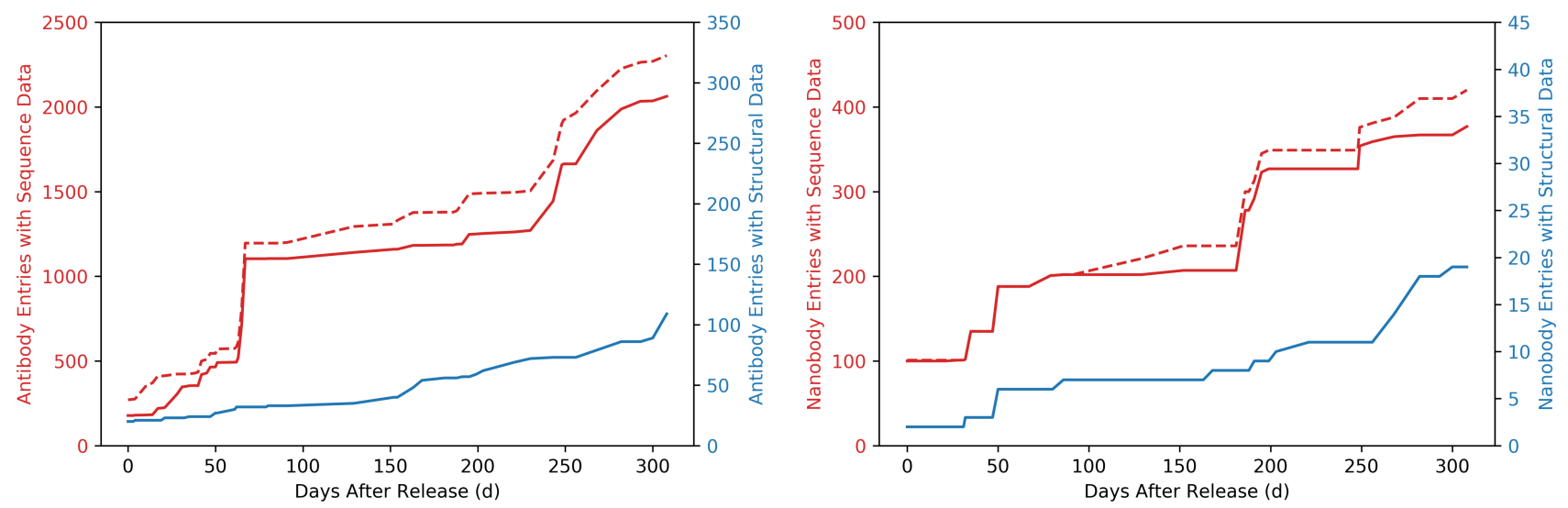

-ேーー CDR3 sequences only

Full sequences

Solved structures

Fig. 1. Comparing the quantity and growth of sequence (red) vs. structural (blue) data referenced by CoV-AbDab for antibodies (left-hand plot) and nanobodies (right-hand plot) against any coronavirus antigen. Structures are classed as solved if evaluated at least once either by X-ray crystallography or cryo-electron microscopy. The $\mathrm{x}$-axis measures the database timestamp after the initial public release of CoV-AbDab on $7^{\text {th }}$ May 2020 (Day 0), up until $11^{\text {th }}$ March 2021 (Day 308 ). CDR3 sequences are often released ahead of full sequences to protect intellectual property during peer review and/or patent filing.

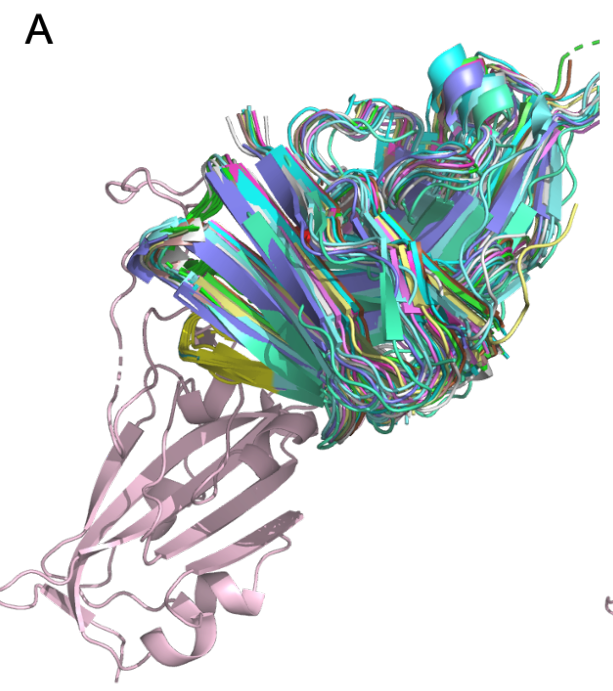

\section{B}

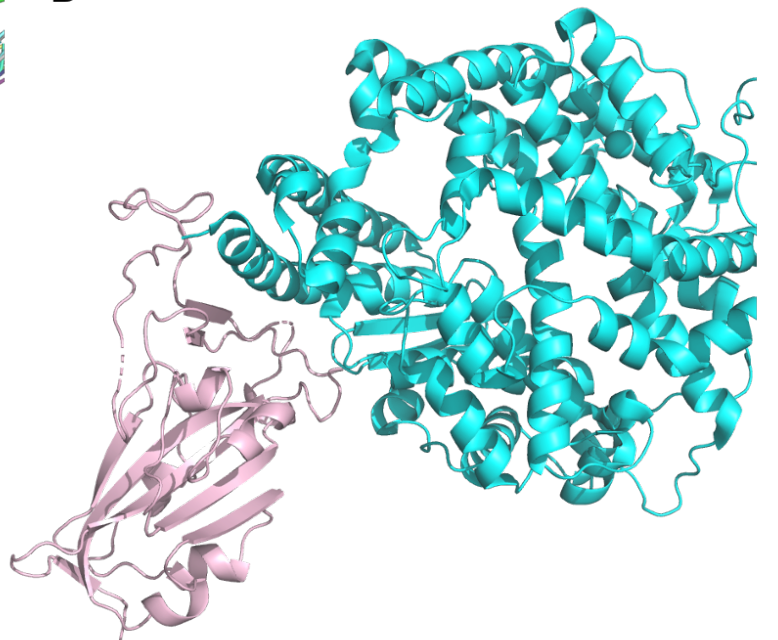

C

\begin{tabular}{l} 
AREAYGMDV \\
\hline ARSPYGGNS \\
\hline AREVAGTYDY \\
\hline ARDVADAFDI \\
\hline ARDFYEGSFDI \\
\hline ARDLGPYGMDV \\
\hline ARDFGDFYFDY \\
ARDYGDYYFDY \\
ARDYGDYYFDY \\
\hline ARDLDVYGLDV \\
ARDLMVYGIDV \\
\hline ARDLGSGDMDV \\
\hline ARDLVVYGMDV \\
\hline ARDLERAGGMDV \\
ARDLGEAGGMDV \\
\hline ARDLDVSGGMDV \\
\hline ARDLQELGSLDY \\
\hline ARVLPMYGDYLDY \\
\hline ARGDVSGYRYGLDY \\
ARGDVSGYRYGLDY \\
ARGDVSGYRYGLDY \\
ARGDVSGYRYGLDY
\end{tabular}

Fig. 2. A) A cartoon representation of the 22 antibodies in the RBD 'neck' cluster binding to the SARS-CoV-2-RBD (salmon) [PDB code $6 X C 2]$ at a similar site to ACE-2. See Table S1 for PDB codes of the 22 antibodies. B) A cartoon representation of ACE-2 (green) binding to SARS-CoV-2 RBD (salmon) [PDB code 6VW1, ACE-2 chain A, SARS-CoV-2-RBD chain E]. C) The CDRH3 sequences represented across the 22 RBD 'neck'-binding antibodies. Lenient VH-clonotypes are separated with solid lines, with the cluster representative highlighted in bold font.

tion (Figure 2A). These antibodies all compete for the ACE2 receptor binding site (Figure 2B). Dejnirattisai et al. identified 13 IGHV3-53/IGHV3-66-derived antibodies engaging this binding site. Even from relatively early in the pandemic, it was clear that a disproportionate number of antibodies reported as able to block ACE-2 binding exploited the IGHV353/IGHV3-66 genes $(1,28,30)$; they appeared significantly more often as binders of this region than would be expected by their abundance in healthy antibody repertoires. Banach $e t$ al. realised that many of the coronavirus-binding antibodies deriving from the IGHV3-53/IGHV3-66 genes possess a conserved set of structural motifs that enable complementarity to a SARS-CoV-2 RBD epitope (55). Our updated analysis reiterates the importance of these $\mathrm{V}$ gene origins in engaging this highly conserved binding site: 19/22 (86\%) of the antibodies align closest to the IGHV3-53 gene, while the remaining 3 align closest to IGHV3-66.

Despite the highly similar V genes, these 22 tightly structurally-clustered antibodies represent 15 different 'lenient VH-only clonotypes' (Figure $3 \mathrm{C}$ ) (clustered antibodies require the same CDRH3 length, 80\% CDRH3 sequence identity, and an identical heavy $\mathrm{V}$ gene, see Methods). This corresponds to 18 'lenient Fv clonotypes' when the light chain is also considered (clustered antibodies must also have the same CDRL3 length, 80\% CDRL3 sequence identity, and an identical light $\mathrm{V}$ gene, see Methods). The analysis of the co-complex structures of these 22 antibodies suggests highly similar functionality, which cannot be wholly identified through clonotyping. Even using lenient clonotype definitions, the antibodies would not be grouped together, so 
A

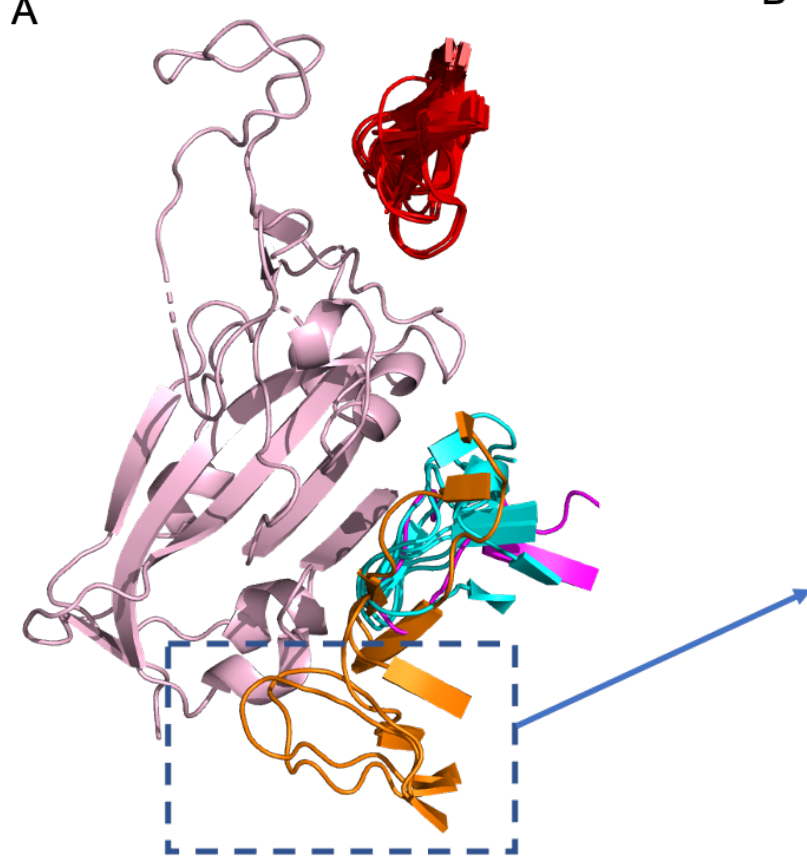

B

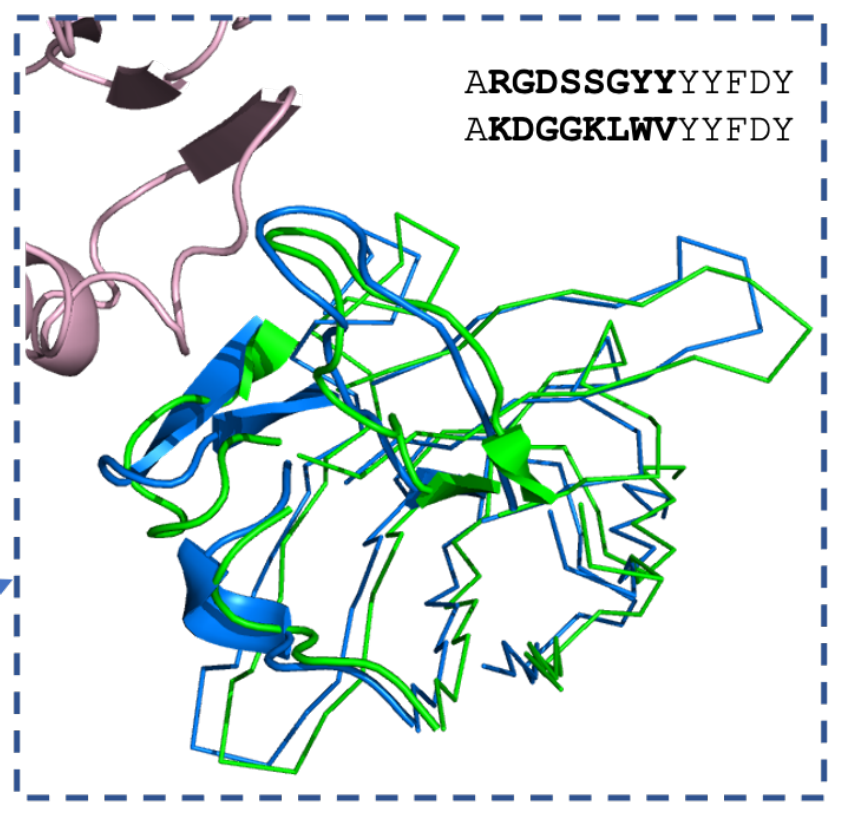

Fig. 3. A) The CDRH3 loops of nanobodies and antibodies of two binding regions, the 'neck' and 'left flank', binding to SARS-CoV-2 RBD (salmon). The 'neck' cluster includes 22 antibodies (red). The 'left flank' region includes five antibodies (the four antibodies identified by (28) are shown in orange, antibody MW06 is shown in magenta) and four nanobodies (shown in blue). MW06 and the nanobodies were not included in the analysis by (28). See Table S1 and Table S2 for antibody and nanobody PDB codes respectively. B) A ribbbon representation of sequence dissimilar antibodies S304 (blue) [PDB code 7L0N] and EY6A (green) [PDB code 6ZER, chain A] binding to the SARS-CoV-2-RBD (salmon) [PDB code 6ZER, chain B]. The CDRH1, CDRH2 and CDRH3 loops are illustrated in cartoon. The CDRH3 sequences of the two antibodies, S304 and EY6A, are shown, with dissimilar residues indicated in bold.

their similar binding mode and functional similarity would be missed.

Dejnirattisai et al. (28) also highlighted an antibody binding cluster located away from the ACE-2 binding site, termed the 'left flank'. This binding region contains four antibodies, EY6A, CR3022, S304 and COVA1-16, (see Methods and Table $\mathrm{S} 1$ for antibody naming conventions). As shown in Figure $3 \mathrm{~A}$, these antibodies appear to bind to slightly different areas. In our updated analysis, we identify one antibody (MW06) and four nanobodies (VHH_U, VHH_V, VHH_W and VHH72) all able bind to the 'left flank' binding region (Figure $3 \mathrm{~A}$ ). We highlight the antibody pair of EY6A and S304, which are structurally similar and adopt a common binding mode to the same RBD epitope (29), but share only $43 \%$ CDRH3 sequence identity (Figure 3B) so could not have been identified as binding to the same site by sequence data alone.

Even across the relatively small number of solved SARSCoV-2 - antibody structures, we can see numerous examples of functionally and structurally similar antibodies that would not be grouped by sequence clustering alone. Grouping coronavirus-binding antibodies into sets that have similar structures therefore represents an orthogonal and promising approach by which to highlight the potential functional commonalities of sequence-dissimilar antibodies.

Paratope analysis. We then analysed the binding interfaces across the set of 48 antibodies, to investigate whether structure needs to be conserved across the whole Fv, or whether conservation over particular regions is typically sufficient.

An average of $\sim 67 \%$ of paratope residues were found to lie on the heavy $(\mathrm{VH})$ chain while $\sim 33 \%$ reside on the light chain (VL). The percentage of paratope residues donated by the most hypervariable region, the CDRH3 loop, varies from just $9 \%$ up to $58 \%$. As expected, structures with longer CDRH3 regions tend to exhibit more $\mathrm{CDRH} 3$-dominated binding; the paratopes of the nine antibodies with CDRH3 length $\geq 19$ on average comprised $41 \%$ CDRH 3 residues. For the 22 highly structurally-conserved antibodies in the RBD neck epitope region, an average of $23 \%$ of the paratope residues originate from CDRH3 (range 8-34\%).

These RBD neck-binding antibodies exhibit high levels of paratope residue conservation across the CDRH1 and CDRH2 (Figure 4), which is also seen at the sequence similarity level. This explains the predominance of the IGHV353/IGHV3-66 genes, as the residues and topologies preencoded by this germline play a key role in neck epitope complementarity. Paratope conservation is considerably less consistent across the $\mathrm{CDRH} 3$ region, accounting for the 18 unique CDRH3 sequences seen across the 22 antibodies. For such epitopes, a clonotyping framework (which conditions on high CDRH3 sequence identity being a pre-requisite for same-epitope binding), will clearly fail to capture the functional similarity of the spectrum of cognate antibodies.

For those antibody paratopes that use CDRH3 sparsely, the paratope is mostly drawn from the CDRH1, CDRH2, CDRL1 and CDRL3 residues, with occasional amino acids provided 
bioRxiv preprint doi: https://doi.org/10.1101/2021.04.12.439478; this version posted April 12, 2021. The copyright holder for this preprint (which was not certified by peer review) is the author/funder, who has granted bioRxiv a license to display the preprint in perpetuity. It is made available under aCC-BY 4.0 International license.
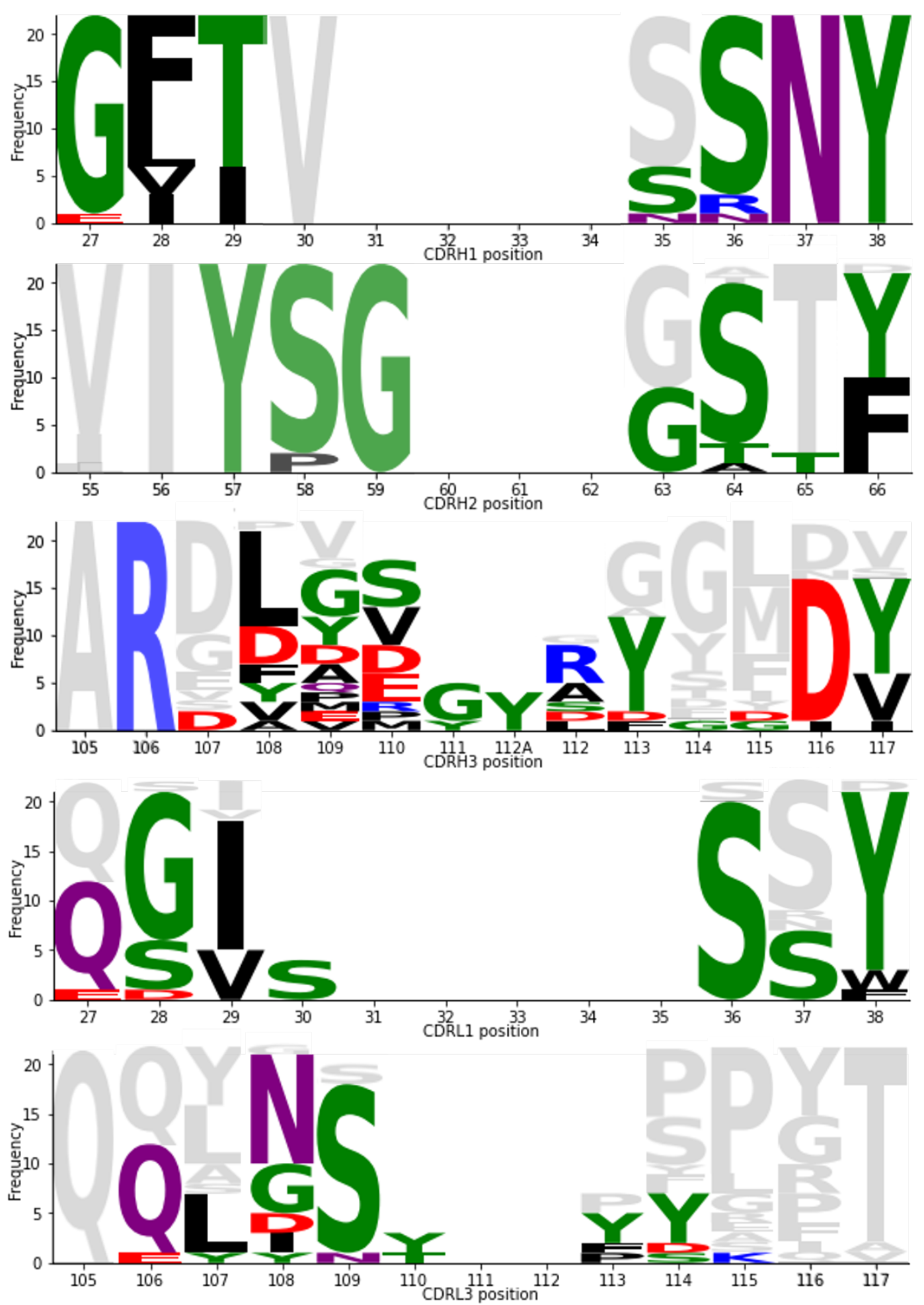

Fig. 4. CDR paratope conservation and divergence across the 22 antibodies in the 'neck' cluster. Frequency indicates the number of times an amino acid was seen at each IMGT-defined CDR position (56). Paratope residues are coloured by side chain chemistry (black $=$ hydrophobic, green $=$ polar neutral, red $=$ acidic, blue $=$ basic, purple $=$

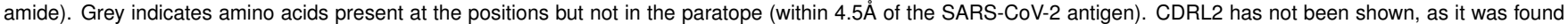
not to contain paratope residues. Produced using Logomaker (57).

by the framework regions (FWRs). In particular, a serine residue at position 83 and/or glycine at position 84 of the light chain (part of the formal FWRL3, IMGT numbering) are disproportionately involved in binding. These residues were found in 18/48 (38\%) of crystallised-antibody paratopes, of which 15 are from the 'neck' structural cluster. Using Arpeggio (58) to identify the type of binding interactions these FWRL 3 residues were involved in, 9 paratopes contained hydrogen bonds between L83 and the RBD, almost always to RBD residue 498. 
Overall, we conclude that structural conservation is important across the entire Fv, to ensure that the paratope residues, which in most coronavirus-binding antibodies are spread evenly throughout the CDRs, are held in equivalent topological positions.

We now perform a comprehensive structure-based analysis on CoV-AbDab, where $95 \%$ of SARS-CoV-2 binding antibodies have no solved structure and $27 \%$ completely lack binding-domain annotation.

\section{Structural Convergence across CoV-AbDab antibod-} ies. As of 11th March, just 5\% $(113 / 2,304)$ of the antibodies in CoV-AbDab had at least one solved X-ray or cryoEM structure, while $~ 90 \%(2,063 / 2,304)$ of the antibodies had full Fv amino acid sequences (Figure 1). We used highthroughput homology modelling approaches to approximate and analyse the geometries of this much broader set of neutralising and non-neutralising antibodies able to bind to multiple coronaviruses, antigens, and domains.

We used ABodyBuilder (59) to homology model the 2,063 antibody entries in $\mathrm{CoV}-\mathrm{AbDab}$ with full $\mathrm{Fv}$ sequences. This resulted in a total of 1,500 models in which every loop was entirely FREAD-modellable without any need for ab initio loop modelling or backbone adjustment (see Methods); we focus on this subset of models as we have the highest confidence in their accuracy $(23,24,59)$. This represents $72.7 \%$ modellability across the set of Fv sequences, a remarkably high percentage relative to recent studies on both healthy and disease-related natural antibody datasets $(23,24)$. Typically, only $\sim 40 \%$ of randomly-sampled human CDRH3s can be homology modelled by FREAD. The increase in modellability is likely to be related to the scientific effort that has gone into solving a large number of SARS-CoV-2-binding antibody structures within the first year of the pandemic. It also hints at a high degree of underlying structural convergence across the reported coronavirus-binding antibodies in $\mathrm{CoV}$ AbDab.

After modelling, we performed structural clustering. Briefly, Fvs with 6 modellable CDRs are first clustered according to their combination of six CDR lengths, and are then further structurally grouped by a greedy clustering algorithm that considers the pairwise structural root-mean square deviation (RMSD) between the selected FREAD template for each CDR region (see Methods for a full description). The result is a set of predicted 'structural clusters', each adopted by at least one $\mathrm{Fv}$ sequence.

The 1,500 homology modelled $\mathrm{Fv}$ regions fell into 1,159 structural clusters, of which 200 were adopted by more than one Fv sequence ('multiple-occupancy' structural clusters). In total, 541/1500 (36.1\%) of the antibodies belonged to a multiple-occupancy structural cluster. For a full breakdown of each multiple-occupancy structural cluster, labelled SC0SC199, see SI Dataset 2.

When applied to an entire antibody repertoire, the number of antibodies with similar structures but different functionalities is likely to be significant (24). However, on 'cleaner' datasets such as CoV-AbDab, where every antibody has been shown to bind a coronavirus antigen (and often a particular domain) in vitro, antibodies with similar $\mathrm{Fv}$ structures should have a high chance of binding to the same surface region and therefore being complementary to the same epitope, as demonstrated in our analysis of solved structures.

Nonetheless, inaccuracy in CDR structure prediction and/or the lack of consideration for paratope residues can lead to misleading clusters of antibodies that have markedly different functionalities. Therefore, to assess (in the context of this large disease-specific antibody database) how predictive belonging to the same structural cluster is of engaging the same epitope, we estimated a pseudo- 'true positive'/'false positive' ratio based on the consistency of the CoV-AbDab epitope metadata across antibodies grouped into the same structural cluster. 'Domain-consistent' structural clusters were classed as those only containing antibodies reported as binding to internally-consistent antigen domains (see Methods).

A total of 184/200 (92\%) of our multiple-occupancy structural clusters were domain-consistent, indicating that structurally clustering with another member of CoV-AbDab is likely to be highly predictive of function.

Amongst the 16 structural clusters considered to be 'false positives', the domain-inconsistent antibody within four structural clusters (SC2, SC84, SC96, SC136) bore significant similarities to at least one other antibody in the cluster, suggesting that some experimentally-deduced epitope labels may be inaccurate. The original papers detailing the binding of the antibodies support this possibility. For example, we classify SC2 as domain-inconsistent, as it contains 8 antibodies that bind to spike outside the $\operatorname{RBD}(60,61)$, and one (COVA2-32 (62)) labelled as binding to the RBD. However, COVA2-32 only marginally met the threshold AUC to be classified as an RBD binder (see Figure 4 of Brouwer $e t$ al. (62)).

The remaining twelve 'false positive' structural clusters are likely to result from a combination of inaccurate structural modelling and/or the fact that bearing the same binding site structure does not guarantee functional commonality. We focus the remainder of our analysis on the $92 \%$ of domainconsistent structural clusters.

Epitope binning. Some members of a structural cluster can have a lower resolution of functional characterisation than others. In these cases, functional properties of the less wellcharacterised antibodies can be inferred from other antibodies predicted to adopt the same structure.

Thirty-one antibodies experimentally shown only to bind to the whole spike protein, or to bind the spike protein but not the RBD, can be localised to a more precise epitope using our structural clusters. For example, three of the antibodies assigned to SC11 that were shown to bind the full-length spike protein, but not a soluble RBD protein (60), can be inferred to bind to the $\mathrm{S} 2$ domain in the same way as cluster members DH1147 and DH1149 (61). Similarly, CC12.24, previously shown only to bind to the whole SARS-CoV-2 spike protein (36), can be localised to the same binding site in the RBD as C139 (12) and COVOX-45 (28) (SC57).

A further 62 antibodies fall into 19 structural clusters for which no antibody has been resolved as binding to a par- 


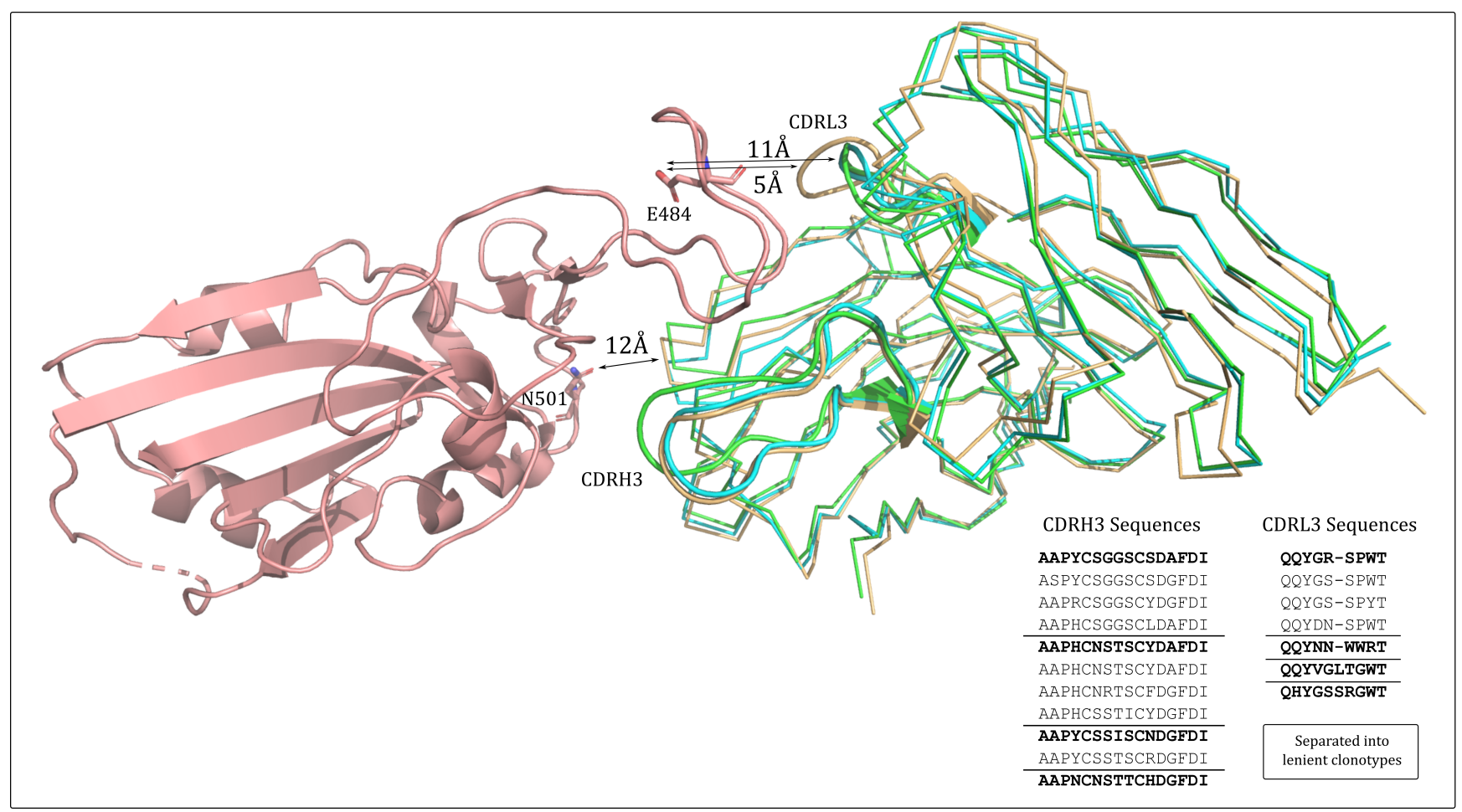

Fig. 5. SARS-CoV-2 RBD-binding antibodies with similar predicted structure that span multiple clonotypes. A representative of structural cluster 3 (SC3, cyan), structural cluster 21 (SC21, orange), and structural cluster 24 (SC24, green) are aligned in the context of the COVOX-253:RBD co-crystal complex (RBD in salmon). The CDRH3 and CDRL3 structures are highlighted in cartoon representation. All 100\% sequence non-redundant CDRH3 and CDRL3 sequences across the three structures are listed, grouped by lenient VH- or VL-clonotype, with the cluster representative in bold font. The alignment shows the various CDRH3 and CDRL3 structures are likely to be topologically compatible with this RBD epitope. Residues E484 and N501, commonly mutated in SARS-CoV-2 variants of concern, are highlighted as sticks and coloured by default atom type. Closest heavy-atom distances between the functional group (carboyxlate/amide heteroatom) and the different structural classes of antibody are shown.

ticular domain. For some structures, the selected FREAD templates could offer an indication of epitope specificity: the PDB structure '6nb8' (63) is used to model all three light chain CDRs in at least one antibody assigned to SC6, SC15, and SC71, while '7ben' (28) is used to model the CDRH3 in all four antibodies assigned to SC16. The antibody in both of these PDB structures engages the SARS-CoV-2 RBD, which could imply that the cluster groups RBD-complementary antibodies. Even ignoring these indications, just 19 binding characterisation experiments could lend functional annotations to over three-times the number of CoV-AbDab entries (62).

Evolutionarily-conserved epitope topologies across coronaviruses. Evolutionarily-conserved epitope topologies are implied by our structural clusters that contain antibodies able to bind multiple coronaviruses. As an extreme example, SC0 pools together a set of 13 IGHV1-69-derived antibodies, of which at least one which has been shown to engage each of SARS-CoV-1, SARS-CoV-2, HKU1, 229E, NL63, OC43, and MERS-CoV. More broadly, 69/184 (37.5\%) of our structural clusters contain an antibody shown on the current levels of data to have cross-coronavirus binding potential. This number may be an underestimate, as several antibodies have only been tested against a single coronavirus strain in vitro.

These epitopes could represent sites of particular vulnerability across coronaviruses; antigen regions whose structure must be preserved for viral function. They therefore reflect the most promising regions against which to design pan- coronavirus neutralising antibodies, and could be exploited in epitope-focused vaccine design strategies (64) to achieve a more broadly neutralising response.

The epitope and corresponding paratope residues within these binding sites will differ between lineages, meaning that sequence-based clustering approaches would struggle to spot their functional commonality. Structural clustering can see beyond paratope profiles to capture broader epitope topology conservation via the geometries of their cognate antibodies.

Epitopes targetable by multiple species. A recent study has shown that mouse and human antibodies use a different distribution of CDRH3 structures, which also varies by B-cell maturation stage (23). This can be rationalised by their speciesspecific gene loci having different predetermined structural biases and the fact that negative selection occurs against different self-epitopes. Nevertheless, assuming that each CDR loop can only adopt a finite set of geometries imposed by the loop closure criterion, and that many of the same antigens would be considered pathogenic to both species, there ought to be some structural overlap between human and mouse antibodies and therefore the potential for some epitopes to be targetable by both species. These would be extremely hard to identify by sequence alone, as human and murine gene loci are highly sequence dissimilar.

We identified SC62, which groups human antibody Ab_511E7 (65) alongside the two murine antibodies DK4 \& DK7 (patent CN111978395A), all of which have been shown to bind the SARS-CoV-2 RBD (albeit only weakly in the case 
of Ab_511E7). Even more remarkably, SC174 pools antibodies from different species confirmed to bind to different coronaviruses; in SC174, a human antibody (C131 (12)) that binds the SARS-CoV-2 RBD is grouped with a murine antibody (F26G18 (66)) shown to bind the full-length SARSCoV-1 spike protein. Should F26G18 be confirmed to engage the RBD, and the SC62 antibodies compete for the same epitope, this would show the ability of structural clustering to identify cross-coronavirus epitopes targetable by multiple organisms.

\section{Structural clusters frequently span multiple clonal lineages.} We analysed each of the 184 domain-consistent structural clusters to determine how often the antibodies clustered together belonged to multiple lenient $\mathrm{Fv}$ clonotypes. A total of $88(47.8 \%)$ contained at least one pair of antibodies from different lenient $\mathrm{Fv}$ clonotypes and 73 (39.7\%) of the structural clusters contain at least two lenient VH-only clonotypes. It is clear that antibodies with both heavy and light chains of differing clonality can frequently co-exist within our structural clusters.

Many structural clusters contain at least one pair of antibodies from the same clonotype; this is unsurprising since the 'near-identical sequence, similar function' assumption underpinning clonotyping experiments is often correct. However, the high frequency with which we group antibodies spanning several clonotypes into the same structural cluster recapitulates the findings of other papers (8-10), and our earlier analysis on solved structures, that clonotyping cannot group together all antibodies capable of same-epitope engagement. In most cases where multiple clonotypes are found in the same structural cluster, it is due to significant differences in the CDRH3 sequence. However, some clusters such as SC134 (which pools COV2-2490 (60) with H712061+K711727 (61)), align closest to different heavy V (IGHV3-7 vs. IGHV3-30) and light V (IGKV1-5 vs. IGKV1D-16) genes.

'Public' Response Antibodies. 'Public' antibodies, those that are raised independently across multiple individuals against an immunodominant epitope, are of high interest to several fields of research, from vaccinology to drug discovery (6769).

Several studies have already identified public SARS-CoV-2 response antibodies based on convergence towards particular clonotype lineages (12-18), but none have yet considered the fact that antibodies from different lineages can exert similar functions. As demonstrated above, structural clustering enables us to group together clonally-distinct antibodies with a high chance of engaging the same epitope. We therefore examined our structural clusters to reveal functionally similar groups of antibodies from different genetic lineages that have been independently isolated across several different studies (i.e. "public structures").

A striking example of a public structure that spans multiple clonotypes is SC3 (Figure 5). SC3 contains nine antibodies from five independent sources, spanning five lenient Fv-clonotypes. All antibodies align closest to the
IGHV1-58/IGKV2-30 gene transcripts but have sequencediverse CDRH3s all containing a common intra-loop disulfide bridge.

The SC21 and SC24 clusters also map to these genes and contain a disulfide bridge; antibodies assigned to SC21 and SC3 have identical CDR lengths but are predicted to have different CDR structures, while SC24 is necessarily classed as a separate structural cluster to SC3 as its CDRL3 loop is of a different length (10 residues rather than 9).

When aligned to the solved COVOX-253:RBD co-crystal structure, SC3, SC21, and SC24 appear to all be topologically complimentary to the same RBD epitope (Figure 5). The length-10, more protruding CDRL3 loop of SC24 is accommodated by the small G485 residue on the RBD, while the CDRH3s across all three structural clusters protrude to a similar extent towards residues F456-N460 on the RBD. The antibodies mapped to SC21 and SC24 (from an additional three independent sources) comprise an additional three lenient Fv-clonotypes, making a total of eight lenient Fv-clonotypes with potential same-epitope complementarity. This set of similar structures has been observed across eight independent studies indicating that the corresponding epitope is immunodominant. Moreover, none of the antibodies directly engage the carboxylate group of residue E484 (5 $\mathrm{A}$ for length-10 CDRL3s or $11 \AA$ for length-9 CDRL3s, with too acute an angle for hydrogen bonding) nor the amide group of N501 ( $\geq 12 \AA$ for all antibody topologies). This should make them of particular interest as clones that might neutralise both wildtype SARS-CoV-2 and the more recent E484K/N501Ycontaining variants of concern.

Soon after we identified this broad structural cluster, a preprint was released by Schmitz et al. (70) showing that many IGHV1-58-encoded SARS-CoV-2 binding antibodies have highly similar residues at equivalent paratope positions (defined by the S2-E12 crystal structure (71)). On this occasion, the other CDRH3 sequence-diverse antibodies hypothesised to have similar function were shortlisted through inspection of their sequences; they all derive from IGHV1-58 germline and bear the $-\mathrm{C}(\mathrm{X})_{4} \mathrm{C}$ - motif within their $\mathrm{CDRH} 3$ loop ( $\mathrm{X}_{4}$ representing four non-cysteine residues). We have shown that structural modelling and clustering supports the theory that these antibodies are functionally similar, and offers a systematic route to the identification of other sequencediverse clusters of functionally-common antibodies that do not bear such clearly conserved motifs.

\section{Discussion}

Here, we have analysed the solved X-ray crystal structures of antibodies and nanobodies bound to SARS-CoV-2 from the perspective of their structural and paratope conservation, and performed the first analysis of thousands of structural models of coronavirus-binding antibodies reported in over 100 independent literature sources.

We have updated the previously-reported sets of antibodies shown to bind to specific regions of the RBD (28) and demonstrated that the antibodies within these clusters are often structurally similar but sequence dissimilar. For exam- 
ple, a cluster of 22 antibodies from 15 different lenient VH clonotypes all approach the same SARS-CoV-2 RBD 'neck' epitope with a closely-related binding mode. The paratopes of these antibodies are highly conserved across the CDRH1 and $\mathrm{CDRH} 2$ regions (accounting for the strong bias towards IGHV3-53/IGHV3-66 gene origins) while the CDRH3 sequence can diverge substantially in sequence identity. The same phenomenon was observed for two antibodies solved engaging the RBD 'left flank' with a near-identical binding mode but just $43 \%$ CDRH3 sequence identity. These scenarios represent a problem for the functional interpretation of sequence-based clustering approaches such as clonotyping; antibodies that are functionally similar would be binned into different clusters. Inspired by the structural similarity of antibodies that bind to the same epitope, we predicted and clustered the structures of the broad set of antibodies documented in CoV-AbDab (1).

We found that this structural clustering is likely to achieve a very high accuracy of epitope binning. Up to $92 \%$ of multiple-occupancy structural clusters grouped antibodies reported to bind to consistent domains, based on the current levels of metadata in CoV-AbDab. This suggests that we can use this method to predict the epitopes of many as-yet uncharacterised coronavirus binders, as well as prospectively to predict the epitopes of newly-isolated SARS-CoV-2 binding antibodies.

These structural clusters also offer a unique perspective on the data that can not be identified through standard, sequencebased clonotyping. First, they can functionally-associate antibodies that derive from highly distinct clonal lineages; around $40 \%$ of our structural clusters contain at least two antibodies from different lenient VH clonotypes. They also functionally connect disease-response antibodies that originate from different species, of interest in the study of functional crossovers between immune repertoires that exploit different gene loci. Moreover, they can reveal which epitope topologies are likely to be conserved across coronavirus strains, helping to co-ordinate efforts to design prophylactics towards more fruitful sites for pan-coronavirus neutralisation.

Such powerful structure-function relationships are likely only possible due to the collation of clean, high confidence binding data. Our work demonstrates the clear value of building large class-specific databases of antibody-binders against extracellular disease-associated antigens.

In addition to offering functional annotations to as-yet uncharacterised antibodies, these databases could also be used to identify important gaps in structural space. This would ensure that time-consuming experimental structure determination efforts are targeted towards sets of antibodies that yield maximal functional insight. For structural clusters with limited existing epitope knowledge, a central antibody could be selected for structural evaluation with the cognate antigen, enabling the functional annotation of many other antibodies in the same structural cluster. Similarly, structurally modelling the entire database reveals which antibodies cannot currently be accurately modeled and should therefore be prioritised for experimental structure characterisation.
As more coronavirus-binding antibody structures continue to be released to the PDB, the coverage and expected accuracy of the CoV-AbDab homology models will increase accordingly, likely further improving the accuracy of our epitope binning over time. However, solved antibody-pandemic virus structures are not a prerequisite of meaningful epitope binning via predicted structure. Forty-eight of our domain-consistent structural clusters currently connect antibodies that bind outside of the RBD, despite the fact no highquality X-ray structures of antibodies binding outside the SARS-CoV-2 RBD had been solved at the time of this analysis $(\leq 2.5 \AA$ resolution, a requirement for use as an ABodyBuilder template). This indicates that structural clustering is able to draw functional connections between antibodies isolated at the start of a pandemic, even if the number of solved antibody-pandemic virus structures is very low.

Overall, our results show that structural information via computational modeling enhances the picture of diseasecharacteristic convergence across SARS-CoV-2 response antibodies. It is clear that while clonotyping COVID-19 antibody repertoires can offer an indication for which epitopes are public in the response, they risk understating the true levels of same-epitope reactivity across individuals. Accurately capturing the functions of the antibodies raised during the immune response is critical when evaluating vaccine efficacy, both against the injected viral strain and against new variants of concern that may arise and alter the immunogenicity of certain epitopes. Structure prediction and clustering has a crucial role to play alongside clonotyping to yield the maximum functional inference from the vast amount of diseasespecific antibody data available.

\section{Methods}

Database Preparation. The version of CoV-AbDab (1) used throughout this analysis was timestamped to the $11^{\text {th }}$ March 2021. The framework and 6 FREAD $(72,73)$ CDR loop databases, which were used during structural modeling to find suitable homologous templates for each antibody region, were also timestamped to contain the quality-filtered contents of SAbDab (74) on $11^{\text {th }}$ March 2021. Quality filtering restricts templates to those solved by $\mathrm{X}$-ray crystallography, with a resolution $\leq 2.5 \AA$ and a B-factor $<80$.

Numbering Scheme and Region Definitions. IMGT numbering (56) is used throughout the manuscript. IMGT CDR region definitions are used to analyse the solved SARSCoV-2 structures. ABodyBuilder uses North CDR definitions in template selection. The North-defined and IMGT-defined CDR3 region lies between IMGT residue numbers 105 and 117 in both the heavy and light chains, meaning clonotype definitions are consistent regardless of region definition (see Clonotyping).

Solved Co-crystal Structure Analysis. Sixty solved Xray co-crystal structures of antibodies and nanobodies bound to SARS-CoV-2 were downloaded from SAbDab. All antibodies were aligned based on the coordinates of the cognate 
SARS-CoV-2-RBD chain using PyMOL functions. Paratope residues were defined as any antibody residues with a heavy atom with $4.5 \AA$ of an antigen heavy atom. We refer to antibodies by name as referenced in CoV-AbDab, using the nomenclature as set out in the literature from which the antibody was sourced. See Table S1 and Table S2 for the names and corresponding PDB codes of antibodies.

Structural Modeling and Analysis. The 2,063 full variable domain $(\mathrm{Fv})$ sequences in $\mathrm{CoV}-\mathrm{AbDab}$ were submitted to the ABodyBuilder antibody modelling tool (59) with default Environment Substitution Score cutoffs. In the first instance, ABodyBuilder seeks to model antibody CDR regions entirely by homology; i.e. to use the FREAD software $(72,73)$ to identify a CDR structural 'template' likely to be adopted by each of the submitted antibody's CDR sequences, considering backbone dihedral angle compatibility and loop graftability onto the framework template. If no suitable structural template can be found for a CDR sequence, $a b$ initio or hybrid homology/ab initio approaches must be used to predict the loop structure, adding uncertainty to model quality. To ensure high model quality, only the 1,500 models for which ABodyBuilder used FREAD to homology model all six CDR loops were carried forward for structural clustering (59) (these models are available as SI Dataset 1).

Structural Clustering Algorithm. These 1,500 Fvs were first split by their combination of six CDR lengths. For each unique CDR length combination, the first antibody in the list was selected as a cluster centre and every subsequent antibody is fed into the following equation:

$$
\sqrt{\frac{\sum_{X}^{(H 1-H 3, L 1-L 3)} D_{X_{12}}^{2} L_{\mathrm{X}}}{\sum_{X}^{(H 1-H 3, L 1-L 3)} L_{\mathrm{X}}}}
$$

where the sum over $X$ refers to each of the six CDRs, $L_{X}$ is the length of North CDRX, and $\mathrm{D}_{X_{12}}$ is the $\mathrm{C}_{\alpha}$ RMSD between the template used to model CDRX in Fv 1 and $\mathrm{Fv} 2$ respectively. If this formula equates to $\leq 0.75 \AA$, the $\mathrm{Fv}$ is clustered with the first cluster centre, otherwise it is held out for the next round of clustering. Once all the Fvs have been considered relative to the first cluster centre, the algorithm progresses in a greedy fashion to select the next unclustered $\mathrm{Fv}$ region as the second cluster centre. The result is a set of structural cluster centres and associated antibody Fv sequences, where each structural cluster only contains antibodies with six identical CDR lengths. This algorithm is adapted from the final step of Repertoire Structural Profiling (24).

'Domain-Consistent' Structural Clusters. The multipleoccupancy structural clusters were each classified as 'domain-consistent/inconsistent' based on the CoV-AbDab binding metadata of their mapped antibody sequences. For instance, the following examples of structural clusters would each be considered as 'domain-consistent':

1. a structural cluster that only contains antibodies characterised as binding to the same antigen and the same domain (e.g. all shown to bind the RBD of the spike protein).
2. a structural cluster that only contains antibodies characterised as binding to internally-consistent domains (e.g. some antibodies labeled as spike N-Terminal Domain (NTD) binders and others labeled as S1 non-RBD binders; where the S NTD is a subdomain of S1 non-RBD).

3. a structural cluster that contains some antibodies that are characterised as binding to the same domain, and others that bind to the same antigen without domain-level resolution (e.g. 4 antibodies shown to bind the spike RBD, and 2 antibodies shown to bind to the full-length spike protein).

4. a structural cluster that only contains antibodies characterised to bind to the same antigen, but no antibody has domain-level resolution (e.g. 5 antibodies all shown to bind to the full-length spike protein, but none are localised to a particular domain).

The following structural clusters would both be considered 'domain-inconsistent':

1. a structural cluster that contains antibodies shown to bind to different antigens.

2. a structural cluster that contains antibodies that bind to the same antigen, but to inconsistent domains (e.g. 3 antibodies that have been shown to bind the spike RBD and 1 shown to bind the spike $\mathrm{S} 2$ domain).

Clonotyping. Clonotyping was performed using an in-house script. Our lenient VH-clonotyping protocol groups Fvs with matching IGHV genes, the same length CDRH3, and $\geq 80 \%$ CDRH3 sequence identity, while our lenient Fv-clonotyping protocol additionally requires cluster members to have a matching IG[K/L]V gene, the same length CDRL3, and $\geq$ 80\% CDRL3 sequence identity. These are lenient clonotyping threshold conditions by community standards (7), as the CDR3 sequence identity threshold is set to its typical lower bound and there is no requirement for cluster members to map to the same IGHJ/IG[K/L]J gene.

\section{ACKNOWLEDGEMENTS}

This work was supported by a Engineering and Physical Sciences Research Council and Medical Research Council Grant [EP/L016044/1] and further research funding from AstraZeneca and UCB Pharma.

\section{AUTHOR CONTRIBUTIONS}

All authors contributed to the design of the research; SAR and MIJR performed the research; SAR, MIJR, CS, and WKW compiled datasets; SAR, MIJR, and CMD wrote the original manuscript; all authors contributed to reviewing and editing the manuscript.

\section{Bibliography}

1. Matthew IJ Raybould, Aleksandr Kovaltsuk, Claire Marks, and Charlotte M Deane. CoVAbDab: the Coronavirus Antibody Database. Bioinformatics, page btaa739, 2020. doi: 10.1093/bioinformatics/btaa739.

2. Renhong Yan, Yuanyuan Zhang, Yaning Li, Lu Xia, Yingying Guo, et al. Structural basis for the recognition of SARS-CoV-2 by full-length human ACE2. Science, 367(6485):14441448, 2020. doi: 10.1126/science.abb2762.

3. Jinsung Yang, Simon JL Petitjean, Melanie Koehler, Qingrong Zhang, Andra C Dumitru, et al. Molecular interaction and inhibition of SARS-CoV-2 binding to the ACE2 receptor. Nat Commun, 11:4541, 2020. doi: 10.1038/s41467-020-18319-6.

4. Jacob D Galson, Sebastian Schaetzle, Rachael JM Bashford-Rogers, Matthew IJ Raybould, Aleksandr Kovaltsuk, et al. Deep sequencing of B cell receptor repertoires from COVID-19 patients reveals strong convergent immune signatures. Front Immunol, 11:605170, 2020. doi: 10.3389/fimmu.2020.605170.

5. Rafael R De Assis, Aarti Jain, Rie Nakajima, Algis Jasinskas, Jiin Felgner, et al. Analysis of SARS-CoV-2 antibodies in COVID-19 convalescent blood using a coronavirus antigen microarray. Nat Commun, 12:6, 2021. doi: 10.1038/s41467-020-20095-2. 
bioRxiv preprint doi: https://doi.org/10.1101/2021.04.12.439478; this version posted April 12, 2021. The copyright holder for this preprint (which was not certified by peer review) is the author/funder, who has granted bioRxiv a license to display the preprint in perpetuity. It is made available under aCC-BY 4.0 International license.

6. Ping Zhao, Jie Cao, Lan-Juan Zhao, Zhao-Lin Qin, Jin-Shan Ke, et al. Immune responses against SARS-coronavirus nucleocapsid protein induced by DNA vaccine. Virology, 331(1): 128-135, 2005. doi: 10.1016/j.virol.2004.10.016.

7. Laura López-Santibáñez-Jácome, S. Eréndira Avendaño-Vázquez, and Carlos Fabián Flores-Jasso. The Pipeline Repertoire for Ig-Seq Analysis. Front Immunol, page 899, 2019. doi: 10.3389/fimmu.2019.00899.

8. Aleksandr Kovaltsuk, Konrad Krawczyk, Jacob D. Galson, Dominic F. Kelly, Charlotte M. Deane, and Johannes Trück. How B-Cell Receptor Repertoire Sequencing Can Be Enriched with Structural Antibody Data. Front Immunol, 8:1753, 2017. doi: 10.3389/fimmu.2017. 01753.

9. Eve Richardson, Jacob D Galson, Paul Kellam, Dominic F Kelly, Sarah E Smith, et al. A computational method for immune repertoire mining that identifies novel binders from different clonotypes, demonstrated by identifying anti-Pertussis toxoid antibodies. mAbs, 13 (1):1869406, 2021. doi: 10.1080/19420862.2020.1869406.

10. Wing Ki Wong, Sarah A. Robinson, Alexander Bujotzek, Guy Georges, Alan P. Lewis, Jiye Shi, James Snowden, Bruck Taddese, and Charlotte M. Deane. Ab-Ligity: Identifying sequence-dissimilar antibodies that bind to the same epitope. mAbs, 13(1):1873478, 2021. doi: 10.1101/2020.03.24.004051.

11. Cinque Soto, Robin G. Bombardi, Andre Branchizio, Nurgun Kose, Pranathi Matta, et al High frequency of shared clonotypes in human B cell receptor repertoires. Nature, 566: 398-402, 2019. doi: 10.1038/s41586-019-0934-8.

12. Davide F Robbiani, Christian Gaebler, Frauke Muecksch, Julio CC Lorenzi, Zijun Wang et al. Convergent antibody responses to SARS-CoV-2 in convalescent individuals. Nature, 584(7821):437-442, 2020. doi: 10.1038/s41586-020-2456-9.

13. Fan Zhang, Rui Gan, Ziqi Zhen, Xiaoli Hu, Xiang Li, et al. Adaptive immune responses to SARS-CoV-2 infection in severe versus mild individuals. Signal Transduct Target Ther, 5 : 156, 2020. doi: 10.1038/s41392-020-00263-y.

14. Jakob Kreye, S Momsen Reincke, Hans-Christian Kornau, Elisa Sánchez-Sendin, Victor M Corman, et al. A SARS-CoV-2 neutralizing antibody protects from lung pathology in a COVID-19 hamster model. Cell, 183(4):1058-1069, 2020. doi: 10.1016/j.cell.2020.09.049.

15. Georgia Bullen, Jacob D Galson, Pedro Villar, Lien Moreels, Line Ledsgaard, et al. Deep mining of early antibody response in COVID-19 patients yields potent neutralisers and reveals high level of convergence. bioRxiv, 2020. doi: 10.1101/2020.12.29.424711.

16. Aurélien Sokal, Pascal Chappert, Giovanna Barba-Spaeth, Anais Roeser, Slim Fourati, et al. Maturation and persistence of the anti-SARS-CoV-2 memory B cell response. Cell, 184(5): 1201-1213, 2021. doi: 10.1016/j.cell.2021.01.050.

17. Christoph Schultheiß, Lisa Paschold, Donjete Simnica, Malte Mohme, Edith Willscher, et al Next-generation sequencing of $\mathrm{T}$ and $\mathrm{B}$ cell receptor repertoires from COVID-19 patients showed signatures associated with severity of disease. Immunity, 53(2):442-455, 2020. doi: 10.1016/j.immuni.2020.06.024.

18. Zachary Montague, Huibin Lv, Jakub Otwinowski, William S DeWitt, Giulio Isacchini, et al. Dynamics of B-cell repertoires and emergence of cross-reactive responses in COVID-19 patients with different disease severity. arXiv, 2020. doi: arXiv:2007.06762.

19. Sandra C. A. Nielsen, Fan Yang, Ramona A. Hoh, Katherine J. L. Jackson, Katharina Roeltgen, et al. Human B Cell Clonal Expansion and Convergent Antibody Responses to SARSCoV-2. Cell Host Microbe, 28(4):516-525, 2020. doi: 10.1016/j.chom.2020.09.002.

20. Bin Ju, Qi Zhang, Jiwan Ge, Ruoke Wang, Jing Sun, et al. Human neutralizing antibodies elicited by SARS-CoV-2 infection. Nature, 584(7819):115-119, 2020. doi: 10.1038/ s41586-020-2380-z.

21. Meng Yuan, Hejun Liu, Nicholas C Wu, Chang-Chun D Lee, Xueyong Zhu, et al. Structural basis of a shared antibody response to SARS-CoV-2. Science, 369(6507):1119-1123, 2020. doi: 10.1126/science.abd2321.

22. Haitao Xiang, Yingze Zhao, Xinyang Li, Peipei Liu, Longlong Wang, et al. Landscapes and dynamic diversifications of B-cell receptor repertoires in COVID-19 patients. bioRxiv, 2020. doi: 10.1101/2020.12.28.424622.

23. Aleksandr Kovaltsuk, Matthew IJ Raybould, Wing Ki Wong, Claire Marks, Sebastian Kelm, et al. Structural diversity of B-cell receptor repertoires along the B-cell differentiation axis in humans and mice. PLOS Computational Biology, 16(2):e1007636, 2020. doi: 10.1371/ journal.pcbi.1007636.

24. Matthew I. J. Raybould, Claire Marks, Aleksandr Kovaltsuk, Alan P. Lewis, Jiye Shi, and Charlotte M. Deane. Public Baseline and Shared Response Structures Support the Theory of Antibody Repertoire Functional Commonality. PLoS Computat Biol, 17(3):e1008781, 2021. doi: 10.1371/journal.pcbi.1008781.

25. Anthony R Rees. Understanding the human antibody repertoire. MAbs, 12(1):1729683, 2020. doi: 10.1080/19420862.2020.1729683.

26. Bryan Briney, Anne Inderbitzin, Collin Joyce, and Dennis R. Burton. Commonality despite exceptional diversity in the baseline human antibody repertoire. Nature, 566(7744):393397, 2019. doi: 10.1038/s41586-019-0879-y.

27. Rohit Arora and Ramy Arnaout. Private Antibody Repertoires Are Public. bioRxiv, 2020 doi: 10.1101/2020.06.18.159699.

28. Wanwisa Dejnirattisai, Daming Zhou, Helen M Ginn, Helen ME Duyvesteyn, Piyada Supasa, et al. The antigenic anatomy of SARS-CoV-2 receptor binding domain. Cell, 2021. doi: 10.1016/j.cell.2021.02.032.

29. Rui Yin, Johnathan D. Guest, Ghazaleh Taherzadeh, Ragul Gowthaman, Ipsa Mittra, et al. Structural and energetic profiling of SARS-CoV-2 antibody recognition and the impact of circulating variants. bioRxiv, 2021. doi: 10.1101/2021.03.21.436311.

30. Christopher O Barnes, Claudia A Jette, Morgan E Abernathy, Kim-Marie A Dam, Shannon R Esswein, et al. SARS-CoV-2 neutralizing antibody structures inform therapeutic strategies. Nature, 588:682-687, 2020. doi: 10.1038/s41586-020-2852-1.

31. Yan Wu, Feiran Wang, Chenguang Shen, Weiyu Peng, Delin Li, et al. A noncompeting pair of human neutralizing antibodies block COVID-19 virus binding to its receptor ACE2. Science, 368(6496):1274-1278, 2020. doi: 10.1126/science.abc2241.

32. Bryan E Jones, Patricia L Brown-Augsburger, Kizzmekia S Corbett, Kathryn Westerndorf, Julian Davies, et al. The neutralizing antibody, LY-CoV555, protects against SARS-CoV2 infection in non-human primates. Sci Trans/ Med, page eabf1906, 2021. doi: 10.1126/ scitranslmed.abf 1906 .
33. Shuo Du, Yunlong Cao, Qinyu Zhu, Guopeng Wang, Xiaoxia Du, et al. Structures of potent and convergent neutralizing antibodies bound to the SARS-CoV-2 spike unveil a unique epitope responsible for exceptional potency. bioRxiv, 2020. doi: 10.1101/2020.07.09.195263.

34. Sarah Ashley Clark, Lars Eric Clark, Junhua Pan, Adrian Coscia, Lindsay GA McKay, et al. Molecular basis for a germline-biased neutralizing antibody response to SARS-CoV-2. bioRxiv, 2020. doi: 10.1101/2020.11.13.381533.

35. Rui Shi, Chao Shan, Xiaomin Duan, Zhihai Chen, Peipei Liu, et al. A human neutralizing antibody targets the receptor-binding site of SARS-CoV-2. Nature, 584(7819):120-124, 2020. doi: 10.1038/s41586-020-2381-y.

36. Thomas F Rogers, Fangzhu Zhao, Deli Huang, Nathan Beutler, Alison Burns, et al. Isolation of potent SARS-CoV-2 neutralizing antibodies and protection from disease in a small animal model. Science, 369(6506):956-963, 2020. doi: 10.1126/science.abc7520.

37. Hejun Liu, Nicholas $C$ Wu, Meng Yuan, Sandhya Bangaru, Jonathan L Torres, et al. Crossneutralization of a SARS-CoV-2 antibody to a functionally conserved site is mediated by avidity. Immunity, 53(6):1272-1280, 2020. doi: 10.1016/j.immuni.2020.10.023.

38. Nicholas C Wu, Meng Yuan, Hejun Liu, Chang-Chun D Lee, Xueyong Zhu, Sandhya Bangaru, Jonathan L Torres, Tom G Caniels, Philip JM Brouwer, Marit J Van Gils, et al. An alternative binding mode of IGHV3-53 antibodies to the SARS-CoV-2 receptor binding domain. Cell Rep, 33(3):108274, 2020. doi: 10.1016/j.celrep.2020.108274.

39. Nicholas $\mathrm{C}$ Wu, Meng Yuan, Sandhya Bangaru, Deli Huang, Xueyong Zhu, et al. A natural mutation between SARS-CoV-2 and SARS-CoV determines neutralization by a crossreactive antibody. PLoS Pathog, 16(12):e1009089, 2020. doi: 10.1371/journal.ppat.1009089.

40. Nicholas K Hurlburt, Emilie Seydoux, Yu-Hsin Wan, Venkata Viswanadh Edara, Andrew B Stuart, et al. Structural basis for potent neutralization of SARS-CoV-2 and role of antibody affinity maturation. Nat Commun, 11(1):5413, 2020. doi: 10.1038/s41467-020-19231-9.

41. Daming Zhou, Helen ME Duyvesteyn, Cheng-Pin Chen, Chung-Guei Huang, Ting-Hua Chen, et al. Structural basis for the neutralization of SARS-CoV-2 by an antibody from a convalescent patient. Nat Struct Mol Biol, 27(10):950-958, 2020. doi: 10.1038/ s41594-020-0480-y.

42. Edurne Rujas, Iga Kucharska, Yong Zi Tan, Samir Benlekbir, Hong Cui, et al. Multivalency transforms SARS-CoV-2 antibodies into broad and ultrapotent neutralizers. bioRxiv, 2020. doi: $10.1101 / 2020.10 .15 .341636$.

43. Yu Guo, Lisu Huang, Guangshun Zhang, Yanfeng Yao, He Zhou, Shu Shen, Bingqing Shen, Bo Li, Xin Li, Mingjie Chen, et al. A SARS-CoV-2 neutralizing antibody with exceptional spike binding coverage and optimized therapeutic potentials. Research Square (Nature Preprint), 2020. doi: 10.21203/rs.3.rs-78945/v1.

44. Cheolmin Kim, Dong-Kyun Ryu, Jihun Lee, Young-II Kim, Ji-Min Seo, et al. A therapeutic neutralizing antibody targeting receptor binding domain of SARS-CoV-2 spike protein. Nat Commun, 12(1):288, 2021. doi: 10.1038/s41467-020-20602-5.

45. Luca Piccoli, Young-Jun Park, M Alejandra Tortorici, Nadine Czudnochowski, Alexandra C Walls, et al. Mapping neutralizing and immunodominant sites on the SARS-CoV-2 spike receptor-binding domain by structure-guided high-resolution serology. Cell, 183(4):10241042, 2020. doi: 10.1016/j.cell.2020.09.037.

46. Federico Bertoglio, Viola Fühner, Maximilian Ruschig, Philip Alexander Heine, Ulfert Rand, et al. A SARS-CoV-2 neutralizing antibody selected from COVID-19 patients by phage display is binding to the ACE2-RBD interface and is tolerant to known RBD mutations. bioRxiv, 2020. doi: 10.1101/2020.12.03.409318.

47. Jiangdong Huo, Audrey Le Bas, Reinis R Ruza, Helen ME Duyvesteyn, Halina Mikolajek, et al. Neutralizing nanobodies bind SARS-CoV-2 spike RBD and block interaction with ACE2. Nat Struct Mol Biol, 27(9):846-854, 2020. doi: 10.1038/s41594-020-0469-6.

48. Hebang Yao, Hongmin Cai, Tingting Li, Bingjie Zhou, Wenming Qin, et al. A high-affinity RBD-targeting nanobody improves fusion partner's potency against SARS-CoV-2. PLoS Pathog, 17(3):e1009328, 2021. doi: 10.1371/journal.ppat.1009328.

49. Yufei Xiang, Sham Nambulli, Zhengyun Xiao, Heng Liu, Zhe Sang, et al. Versatile and multivalent nanobodies efficiently neutralize SARS-CoV-2. Science, 370(6523):1479-1484, 2020. doi: $10.1126 /$ science.abe 4747 .

50. Daniel Wrapp, Dorien De Vlieger, Kizzmekia S Corbett, Gretel M Torres, Nianshuang Wang, et al. Structural basis for potent neutralization of betacoronaviruses by single-domain camelid antibodies. Cell, 181(5):1004-1015, 2020. doi: 10.1016/j.cell.2020.04.031.

51. Paul-Albert Koenig, Hrishikesh Das, Hejun Liu, Beate M Kümmerer, Florian N Gohr, et al. Structure-guided multivalent nanobodies block SARS-CoV-2 infection and suppress mutational escape. Science, 371(6530):eabe6230, 2021. doi: 10.1126/science.abe6230.

52. Tingting Li, Hongmin Cai, Hebang Yao, Bingjie Zhou, Yapei Zhao, et al. A potent synthetic nanobody targets RBD and protects mice from SARS-CoV-2 infection. BioRxiv, 2020. doi: 10.1101/2020.06.09.143438.

53. Micah Rapp, Yicheng Guo, Eswar Reddy Reddem, Lihong Liu, Pengfei Wang, et al. Modular basis for potent SARS-CoV-2 neutralization by a prevalent $\mathrm{VH} 1$-2-derived antibody class. Cell Rep, 35(1):108950, 2021. doi: 10.1016/j.celrep.2021.108950.

54. Emma C Thomson, Laura E Rosen, James G Shepherd, Roberto Spreafico, Ana da Silva Filipe, et al. Circulating SARS-CoV-2 spike N439K variants maintain fitness while evading antibody-mediated immunity. Cell, 184(5):1171-1187, 2021. doi: 10.1016/j.cell. 2021.01.037.

55. Bailey B. Banach, Gabriele Cerutti, Ahmed S. Fahad, Chen-Hsiang Shen, Matheus Oliveira de Souza, et al. Paired heavy and light chain signatures contribute to potent SARS-CoV-2 neutralization in public antibody responses. bioRxiv, 2021. doi: 10.1101/2020.12.31.424987.

56. M-P. Lefranc, Christelle Pommié, Manuel Ruiz, V Giuducelli, Elodie Foulquier, et al. IMGT unique numbering for immunoglobulin and $T$ cell receptor variable domains and Ig superfamily V-like domains. Dev Comp Immunol, 27(1):55-77, 2003. doi: 10.1016/S0145-305X(02) 00039-3.

57. Ammar Tareen and Justin B Kinney. Logomaker: beautiful sequence logos in Python. Bioinformatics, 36(7):2272-2274, 2020. doi: 10.1093/bioinformatics/btz921.

58. Harry C Jubb, Alicia P Higueruelo, Bernardo Ochoa-Montaño, Will R Pitt, David B Ascher, et al. Arpeggio: a web server for calculating and visualising interatomic interactions in protein structures. J Mol Biol, 429(3):365-371, 2017. doi: 10.1016/j.jmb.2016.12.004.

59. Jinwoo Leem, James Dunbar, Guy Georges, Jiye Shi, and Charlotte M. Deane. ABodyBuilder: Automated antibody structure prediction with data-driven accuracy estimation. 
bioRxiv preprint doi: https://doi.org/10.1101/2021.04.12.439478; this version posted April 12, 2021. The copyright holder for this preprint (which was not certified by peer review) is the author/funder, who has granted bioRxiv a license to display the preprint in perpetuity. It is made available under aCC-BY 4.0 International license.

mAbs, 8(7):1259-1268, 2016. doi: 10.1080/19420862.2016.1205773.

60. Seth J. Zost, Pavlo Gilchuk, Rita E. Chen, James Brett Case, Joseph X. Reidy, et al. Rapid isolation and profiling of a diverse panel of human monoclonal antibodies targeting the SARS-CoV-2 spike protein. Nat Med, 26:1422-1427, 2020. doi: 10.1038/ s41591-020-0998-x.

61. Dapeng Li, Robert J Edwards, Kartik Manne, David R. Martinez, Alexandra Schäfer, et al. The functions of SARS-CoV-2 neutralizing and infection-enhancing antibodies in vitro and in mice and nonhuman primates. bioRxiv, 2021. doi: 10.1101/2020.12.31.424729.

62. Philip JM Brouwer, Tom G Caniels, Karlijn van der Straten, Jonne L Snitselaar, Yoann Aldon, et al. Potent neutralizing antibodies from COVID-19 patients define multiple targets of vulnerability. Science, 369(6504):643-650, 2020. doi: 10.1126/science.abc5902.

63. Dora Pinto, Young-Jun Park, Martina Beltramello, Alexandra C Walls, M Alejandra Tortorici, et al. Cross-neutralization of SARS-CoV-2 by a human monoclonal SARS-CoV antibody. Nature, 583(7815):290-295, 2020. doi: 10.1038/s41586-020-2349-y.

64. Bruno E. Correia, John T. Bates, Rebecca J. Loomis, Gretchen Baneyx, Chris Carrico, et al. Proof of principle for epitope-focused vaccine design. Nature, 507:201-206, 2014. doi: 10.1038/nature12966.

65. Xiaojian Han, Yingming Wang, Shenglong $\mathrm{Li}$, Chao Hu, Tingting $\mathrm{Li}$, et al. A rapid and efficient screening system for neutralizing antibodies and its application for the discovery of potent neutralizing antibodies to SARS-CoV-2 S-RBD. bioRxiv, 2020. doi: 10.1101/2020.08. 19.253369.

66. Michael J. Gubbins, Frank A. Plummer, Xin Y. Yuan, Darrell Johnstone, Mike Drebot, et al. Molecular characterization of a panel of murine monoclonal antibodies specific for the SARS-coronavirus. Mol Immunol, 42(1):125-136, 2005. doi: https://doi.org/10.1016/j. molimm.2004.06.032.

67. Ian Setliff, Wyatt J. McDonnell, Nagarajan Raju, Robin G. Bombardi, Amyn A. Murji, et al. Multi-Donor Longitudinal Antibody Repertoire Sequencing Reveals the Existence of Public Antibody Clonotypes in HIV-1 Infection. Cell Host Microbe, 26(3):845-854, 2018. doi: 10.1016/j.chom.2018.05.001.

68. David Forgacs, Rodrigo B. Abreu, Giuseppe A. Sautto, Greg A. Kirchenbaum, Elliott Drabek, et al. Convergent antibody evolution and clonotype expansion following influenza virus vaccination. PLOS ONE, 16(2):e0247253, 2021. doi: 10.1371/journal.pone.0247253.

69. Claire Marks and Charlotte M. Deane. How antibody repertoire data are changing antibody science. J Biol Chem, 295(25):9823-9837, 2021. doi: 10.1074/jbc.REV120.010181.

70. Aaron J. Schmitz, Jackson S. Turner, Zhuoming Liu, Ishmael D. Aziati, Rita E. Chen, et al. A public vaccine-induced human antibody protects against SARS-CoV-2 and emerging variants. bioRxiv, 2021. doi: 10.1101/2021.03.24.436864.

71. M. Alejandra Tortorici, Martina Beltramello, Florian A. Lempp, Dora Pinto, Ha V. Dang, et al. Ultrapotent human antibodies protect against SARS-CoV-2 challenge via multiple mechanisms. Science, 370(6519):950-957, 2020. doi: 10.1126/science.abe3354.

72. Yoonjoo Choi and Charlotte M. Deane. FREAD revisited: Accurate loop structure prediction using a database search algorithm. Proteins, 78(6):1431-1440, 2010. doi: 10.1002/prot. 22658.

73. Yoonjoo Choi and Charlotte M. Deane. Predicting antibody complementarity determining region structures without classification. Mol BioSyst., 12(7):3327-3334, 2011. doi: 10.1039/ $\mathrm{c} 1 \mathrm{mb} 05223 \mathrm{c}$.

74. James Dunbar, Konrad Krawczyk, Jinwoo Leem, Terry Baker, Angelika Fuchs, et al. SAbDab: The structural antibody database. Nucleic Acids Research, 42(D1), 2014. doi: 10.1093/nar/gkt1043.

75. Jan Ter Meulen, Edward N Van Den Brink, Leo LM Poon, Wilfred E Marissen, Cynthia SW Leung, et al. Human monoclonal antibody combination against SARS coronavirus: synergy and coverage of escape mutants. PLoS Med, 3(7):e237, 2006. doi: 10.1371/journal.pmed. 0030237.

76. Emilie Seydoux, Leah J Homad, Anna J MacCamy, K Rachael Parks, Nicholas K Hurlburt, et al. Characterization of neutralizing antibodies from a SARS-CoV-2 infected individual. bioRxiv, 2020. doi: 10.1101/2020.05.12.091298.

77. Shane Miersch, Zhijie Li, Reza Saberianfar, Mart Ustav, James Brett Case, et al. Tetravalent SARS-CoV-2 Neutralizing Antibodies Show Enhanced Potency and Resistance to Escape Mutations. bioRxiv, 2020. doi: 10.1101/2020.10.31.362848.

78. Lihong Liu, Pengfei Wang, Manoj S Nair, Jian Yu, Micah Rapp, et al. Potent neutralizing antibodies against multiple epitopes on SARS-CoV-2 spike. Nature, 584(7821):450-456, 2020. doi: 10.1038/s41586-020-2571-7.

79. J. Wang, S. Jiao, R. Wang, J. Zhang, M. Zhang, et al. Crystal structure of SARS-CoV-2 Spike RBD in complex with MW06 Fab. 2020. doi: 10.2210/pdb7DPM/pdb.

80. X. Wang, L. Zhang, J. Ge, and R. Wang. Crystal structure of SARS-CoV-2 antibody P2C1F11 and RBD. 2020. doi: 10.2210/pdb7CDI/pdb

81. X. Wang and J. Ge. Crystal structure of SARS-CoV-2 antibody P2C-1A3 and RBD. 2020. doi: $10.2210 / \mathrm{pdb} 7 \mathrm{CDJ} / \mathrm{pdb}$. 


\begin{tabular}{|c|c|c|c|}
\hline Ab Name & PDB Code & Citation & Epitope Cluster \\
\hline B38 & $7 \mathrm{BZ5}$ & (31) & Neck \\
\hline Bamlanivimab (LY-CoV555) & $7 \mathrm{KMG}$ & (32) & *Left Shoulder \\
\hline BD-236 & $7 \mathrm{CHB}$ & (33) & Neck \\
\hline BD-368-2 & $7 \mathrm{CHC}$ & (33) & Left Shoulder \\
\hline BD-604 & 7CHF & (33) & Neck \\
\hline BD-629 & 7CH5 & (33) & Neck \\
\hline C102 & $7 \mathrm{~K} 8 \mathrm{M}$ & (12) & *Neck \\
\hline C1A-B3 & $7 \mathrm{KFW}$ & (34) & *Neck \\
\hline C1A-B12 & $7 \mathrm{KFV}$ & (34) & *Neck \\
\hline $\mathrm{C} 1 \mathrm{~A}-\mathrm{C} 2$ & $7 \mathrm{KFX}$ & (34) & *Neck \\
\hline C1A-F10 & $7 \mathrm{KFY}$ & (34) & *Neck \\
\hline CB6 & 7C01 & (35) & Neck \\
\hline $\mathrm{CC} 12.1$ & $6 \mathrm{XC} 2$ & (36) & Neck \\
\hline $\mathrm{CC} 12.3$ & $6 \mathrm{XC} 4$ & (36) & Neck \\
\hline COVA1-16 & 7JMW & $(37,62)$ & Left Flank \\
\hline COVA2-04 & 7JMO & $(38,62)$ & Neck \\
\hline COVA2-39 & 7JMP & $(38,62)$ & Right Shoulder \\
\hline COVOX-45 & 7BEL & (28) & Right Flank \\
\hline COVOX-75 & $7 \mathrm{BEO}$ & (28) & Right Shoulder \\
\hline COVOX-88 & 7BEL & (28) & Left Shoulder \\
\hline COVOX-150 & 7BEI & (28) & Neck \\
\hline COVOX-158 & 7BEJ & (28) & Neck \\
\hline COVOX-253 & 7BEN & (28) & Left Shoulder \\
\hline COVOX-253H55L & 7BEO & (28) & Left Shoulder \\
\hline COVOX-269 & 7BEM & (28) & Neck \\
\hline COVOX-316 & $7 \mathrm{BEH}$ & (28) & Left Shoulder \\
\hline COVOX-384 & 7BEP & (28) & Left Shoulder \\
\hline CR3022 & 7JN5 & $(39,75)$ & Left Flank \\
\hline CV07-250 & $6 \mathrm{XKQ}$ & (14) & Right Shoulder \\
\hline CV07-270 & $6 \mathrm{XKP}$ & (14) & Left Shoulder \\
\hline CV30 & 6XE1 & $(40,76)$ & Neck \\
\hline EY6A & 6ZER & (41) & Left Flank \\
\hline Fab_15033-7 & $7 \mathrm{KLH}$ & (77) & *Right Shoulder \\
\hline Fab-52 & $7 \mathrm{~K} 9 \mathrm{Z}$ & (42) & *Left Shoulder \\
\hline Fab-298 & $7 \mathrm{~K} 9 \mathrm{Z}$ & (42) & *Right Shoulder \\
\hline Fab2-15 & 7L5B & $(53,78)$ & *Shoulder \\
\hline LY-CoV481 & 7KMI & $(32)$ & Neck \\
\hline LY-CoV488 & $7 \mathrm{KMH}$ & (32) & Neck \\
\hline MW06 & 7DPM & (79) & $*$ Left Flank \\
\hline P4A1 & $7 \mathrm{CJF}$ & (43) & Neck \\
\hline P2B-2F6 & $7 \mathrm{BWJ}$ & (20) & Left Shoulder \\
\hline P2C-1F11 & 7CDI & (80) & *Neck \\
\hline $\mathrm{P} 2 \mathrm{C}-1 \mathrm{~A} 3$ & 7CDJ & (81) & *Shoulder \\
\hline Regdanvimab & 7CM4 & (44) & *Right Shoulder \\
\hline S2H14 & 7JX3 & (45) & Right Shoulder \\
\hline S304 & $7 \mathrm{LON}$ & $(45,63)$ & Left Flank \\
\hline S309 & $7 \mathrm{JX} 3$ & $(45,63)$ & Right Flank \\
\hline STE90-C11 & 7B30 & (46) & *Neck \\
\hline
\end{tabular}

\section{SI Tables}

Table S1. Antibodies with a solved crystal structures in complex with a SARS-CoV-2 antigen. Antibodies were assigned to their nearest epitope cluster, as defined by Dejnirattisai et al. (28). Antibodies whose epitope cluster is labelled with a star $(*)$ were not included in the previous analysis by Dejnirattisai et al. (28). The new cluster label 'Shoulder' indicates that antibody's epitope lies between the left and right shoulder clusters. 
bioRxiv preprint doi: https://doi.org/10.1101/2021.04.12.439478; this version posted April 12, 2021. The copyright holder for this preprint (which was not certified by peer review) is the author/funder, who has granted bioRxiv a license to display the preprint in perpetuity. It is made available under aCC-BY 4.0 International license.

\begin{tabular}{|l|r|r|r|}
\hline Nb Name & PDB Code & Citation & Epitope Cluster \\
\hline H11-D4 & 6YZ5 & $(47)$ & *Left Shoulder \\
H11-H4 & 6ZBP & $(47)$ & *Left Shoulder \\
MR17 & 7C8W & $48,52)$ & *Shoulder \\
MR17_K99Y & 7CAN & $(52)$ & *Shoulder \\
Nb20 & 7JVB & $(49)$ & *Shoulder \\
SR4 & 7C8V & $(52)$ & *Left Shoulder \\
SR31 & 7D2Z & $(52)$ & *Left Shoulder \\
VHH-72 & $6 \mathrm{WAQ}$ & $(50)$ & *Left Flank \\
VHH_E & 7KN5 & $(51)$ & Left Shoulder \\
VHH_U & 7KN5 & $(51)$ & *Left Flank \\
VHH_V & 7KN6 & $(51)$ & *Left Flank \\
VHH_W & 7KN7 & $(51)$ & *Left Flank \\
\hline
\end{tabular}

Table S2. Nanobodies with a solved crystal structures in complex with a SARS-CoV-2 antigen. nanobodies were assigned to their nearest epitope cluster, as defined by Dejnirattisai et al. (28). Nanobodies whose epitope cluster is labelled with a star (*) were not included in the previous analysis by Dejnirattisai et al. (28). The new cluster label 'Shoulder' indicates that antibody's epitope lies between the left and right shoulder clusters. 